\title{
MR lung perfusion measurements in adolescents after congenital diaphragmatic hernia: correlation with spirometric lung function tests
}

\author{
Verena Groß ${ }^{1} \cdot$ Katrin Zahn $^{2} \cdot$ Kristina Maurer $^{2} \cdot$ Lucas Wessel $^{2} \cdot$ Thomas Schaible $^{3} \cdot$ Stefan O. Schoenberg ${ }^{1}$. \\ Christel Weiß $\beta^{4} \cdot$ Frank G. Zoellner $^{5} \cdot$ Meike Weis $^{1}$ (i)
}

Received: 15 May 2021 / Revised: 1 August 2021 / Accepted: 1 September 2021 / Published online: 6 November 2021

(c) The Author(s) 2021, corrected publication 2022

\begin{abstract}
Objectives To evaluate whether lung perfusion continues to be reduced in 10-year-old children after congenital diaphragmatic hernia $(\mathrm{CDH})$ and whether lung perfusion values correlate with spirometric lung function measurements.

Methods Fifty-four patients after CDH repair received dynamic contrast-enhanced (DCE) magnetic resonance imaging (MRI)-based lung perfusion measurements at the age of 10 years (10.2 \pm 1.0 years). Additionally, a control group of 10 children has been examined according to the same protocol. Lung spirometry was additionally available in 43 patients of the $\mathrm{CDH}$ group. A comparison of ipsilateral and contralateral parameters was performed.

Results Pulmonary blood flow (PBF) was reduced on the ipsilateral side in CDH patients $(60.4 \pm 23.8$ vs. $93.3 \pm 16.09 \mathrm{~mL} / 100 \mathrm{~mL} / \mathrm{min} ; p<0.0001)$. In comparison to the control group, especially the ratio of ipsilateral to contralateral, PBF was reduced in $\mathrm{CDH}$ patients $(0.669 \pm 0.152$ vs. $0.975 \pm 0.091 ; p<0.0001)$. There is a positive correlation between ipsilateral pulmonary blood flow, and spirometric forced 1 -s volume $(r=0.45 ; p=0.0024)$.

Conclusions Pulmonary blood flow impairment persists during childhood and correlates with spirometric measurements. Without the need for ionizing radiation, MRI measurements seem promising as follow-up parameters after CDH.

Key Points

- Ten-year-old children after congenital diaphragmatic hernia continue to show reduced perfusion of ipsilateral lung.

- Lung perfusion values correlate with lung function tests after congenital diaphragmatic hernia.
\end{abstract}

Keywords Perfusion imaging $\cdot$ Hernias, diaphragmatic, congenital $\cdot$ Magnetic resonance imaging $\cdot$ Lung $\cdot$ Spirometry

$\begin{array}{ll}\text { Abbreviations } \\ \text { CDH } & \text { Congenital diaphragmatic hernia } \\ \text { ECMO } & \begin{array}{l}\text { Extracorporeal membrane oxygenation } \\ \text { therapy }\end{array} \\ \text { FEV1 } & \text { Forced expiratory volume per second }\end{array}$

Verena Groß and Katrin Zahn are joint-first, equal contribution.

Meike Weis

meike.weis@medma.uni-heidelberg.de

1 Department of Clinical Radiology and Nuclear Medicine, University Medical Center Mannheim, Heidelberg University, Theodor-Kutzer-Ufer 1-3, 68167 Mannheim, Germany

2 Department of Pediatric Surgery, University Medical Center Mannheim, Heidelberg University, Theodor-Kutzer-Ufer 1-3, 68167 Mannheim, Germany

3 Department of Neonatology, University Medical Center Mannheim, Heidelberg University, Theodor-Kutzer-Ufer 1-3, 68167 Mannheim, Germany

$\begin{array}{ll}\text { FVC } & \text { Forced vital capacity } \\ \text { GRAPPA } & \begin{array}{l}\text { Generalized autocalibrating partially parallel } \\ \text { acquisition }\end{array} \\ \text { MRI } & \text { Magnetic resonance imaging } \\ \text { MTT } & \text { Mean transit time } \\ \text { PBF } & \text { Pulmonary blood flow }\end{array}$

4 Department of Medical Statistics and Biomathematics, University Medical Center Mannheim, Heidelberg University, Theodor-Kutzer-Ufer 1-3, 68167 Mannheim, Germany

5 Computer Assisted Clinical Medicine, Mannheim Institut for Intelligent System, Medical Faculty Mannheim, Heidelberg University, Heidelberg University, Theodor-Kutzer-Ufer 1-3, 68167 Mannheim, Germany 
PBV Pulmonary blood volume

TWIST Time-resolved angiography with stochastic trajectories

\section{Introduction}

Advances in understanding and treatment of congenital diaphragmatic hernia (CDH) have led to increased survival rates [1]. Nevertheless, this gain is associated with an increased degree of morbidity of survivors. This brings up the need for structured follow-up programs to recognize and potentially treat children after $\mathrm{CDH}$. Focus on follow-up after $\mathrm{CDH}$ can also be observed in the increasing number of publications dealing with this topic.

Jesselstijn et al. published a survey of the $\mathrm{CDH}$ consortium members, which dealt with the question of how followup takes place in their hospital: There is a wide diversity concerning time-point and type of examination [2].

Despite differences in follow-up scheme, members of $\mathrm{CDH}$ consortium agree that follow-up needs to focus on the main problems children after $\mathrm{CDH}$ have, namely, feeding problems, developmental retardation in different areas of life, and lung function impairment. Additionally, the potential hernia recurrence has to be evaluated [2-4]. Most lung function measurements depend on the compliance of the child and are therefore limited in younger children. One image modality to measure lung function parameters is scintigraphy, which needs the application of ionizing radiation.

With magnetic resonance imaging (MRI), lung perfusion can be measured without ionizing radiation. Previous studies based on MRI perfusion measurements demonstrated for 2-year-old children that after $\mathrm{CDH}$ they show reduced values on the ipsilateral side. Additionally, 2-year-old children after ECMO requirement show even more reduced values [5-7]. It has not been evaluated whether ipsilateral perfusion defects persist during childhood and whether they correlate with lung function measurements.

Therefore, this study aimed to measure MR lung perfusion of adolescent children after $\mathrm{CDH}$ and correlate them with spirometric lung function measurements.

\section{Materials and methods}

\section{Patients}

Inclusion criteria for $\mathrm{CDH}$ patients for the study evaluation were (i) taking part in a local follow-up protocol), which includes MR lung perfusion measurements at the age of 10 to 12 years (10.2 \pm 1.0 years); and (ii) sufficient image quality. Consequently, 54 patients ( 30 males, 24 females) could be included into this study. A total of $81.5 \%$ of patients suffered initially from left-sided and $18.5 \%$ from right-sided $\mathrm{CDH}$. ECMO was required by $40.7 \%$ during the neonatal period.

Additionally, 10 controls ( 3 males, 7 females) without $\mathrm{CDH}$ have been included in this study (inflammation, $n=4$; tumor, $n=3$; neurological disorder, $n=2$; trauma $n=1$ ). Inclusion criteria for control group were (i) correct imaging protocol, (ii) no disease affecting lung perfusion, and (iii) correct patient age-which means an age interval of 10 to 12 years. The mean age of the control group was $11.4 \pm 1.0$ years. Perfusion imaging was included into the imaging protocol to visualize the cervical and thoracic vascular anatomy.

Local research ethics committee approved retrospective evaluation of study cohort and analysis of control group, respectively.

\section{MR imaging}

All MR examinations have been performed on a single 3-T MRI system (Magnetom TimTrio, Siemens Healthineers). Lung perfusion imaging was included in the MR protocol, which consists of a head and thoracic examination. The entire examination took 25-30 min, $15 \mathrm{~min}$ of which for the thorax. A combination of coils (head, neck, and body phased-array) was used. No sedation was necessary.

A three-dimensional time-resolved angiography with stochastic trajectories (TWIST) sequence was applied for perfusion imaging [7]. TWIST view-sharing was set to $15 \%$ sampling density in the central and 20\% in the outer region. Echo time was $0.78 \mathrm{~ms}$ and repetition time $2.28 \mathrm{~ms}$ with a flip angle of $14^{\circ}$. Generalized autocalibrating partially parallel acquisition (GRAPPA) of factor 3 was used. A temporal resolution of $1.5 \mathrm{~s}$ was chosen with an isotropic spatial resolution of $2 \mathrm{~mm}$. Contrast agent (Dotarem, Guerbet) with a dosage of $0.05 \mathrm{mmol} / \mathrm{kg}$ body weight, diluted with the same volume of sodium chloride and followed by a sodium chloride bolus of $10 \mathrm{~mL}$, was administered at a flow rate of $1 \mathrm{~mL} / \mathrm{s}$ after the acquisition of five baseline images. Perfusion imaging was obtained under free breathing.

\section{Data analysis}

Perfusion was quantified using a pixel-by-pixel deconvolution approach, which is implemented in an in-house-developed and certified OsiriX plugin [8,9]. The arterial input function (AIF) was derived by a region of interest (ROI) placed in the main stem of the pulmonary artery. As quantitative pulmonary perfusion parameters, the pulmonary blood flow (PBF), the pulmonary blood volume (PBV), and the mean transit time (MTT) were calculated. The whole lung was manually delineated based on TWIST sequences and consequently a ROI that included whole lung has been 
calculated. Macrovessels of the hilar region have been excluded from the analysis. By applying this ROI on perfusion maps, mean values of PBF, PBV, and MTT per lung side could be calculated. Lung delineation was performed by two readers ( 1 year and 6 years experience in lung MRI) in agreement. The applied method has already demonstrated good inter- and intra-reader agreement in a previous study [5].

\section{Statistical analysis}

All statistical calculations and graphical representations of data in this study were performed with a dedicated software (GraphPad Prism Version 8.3.0, GraphPad Software and SAS, SAS Institute Inc.). Lung perfusion parameters (PBF, PBV, MTT) of the ipsilateral versus contralateral side were compared and proven for significance by a paired $t$-test. Furthermore, differences in lung perfusion parameters were tested between the $\mathrm{CDH}$ group vs. control group, children with vs. without need for ECMO therapy, and children with vs. without clinically proven respiratory disorder. All of these comparisons were quantified and proven for significance by an unpaired $t$-test. The results of the spirometric lung function measurements were compared between children with the need for ECMO therapy and those without. This comparison was also quantified by an unpaired $t$-test.

Pearson's correlation analysis was performed to detect a linear correlation between MRI-based lung perfusion parameters and spirometric measurements.
For all statistical tests mentioned above, a normal distribution was assumed, which was tested by the Shapiro-Wilk test.

In all tests, $p<0.05$ was considered statistically significant.

\section{Lung function measurement}

Spirometric measurements of $\mathrm{CDH}$ patients were performed on the same day as the MR examination. Due to retrospective approach of lung perfusion analysis, the control group did not receive lung function measurements. Results included the following values: forced expiratory volume per second (FEV1), forced vital capacity (FVC), Tiffeneau Index, which is the ratio of FEV1 and FVC, as well as the type of lung function impairment (restrictive, obstructive, combined, none). FEV1, FVC, and Tiffeneau Index were calculated in percentage of the nominal value and absolute terms.

\section{Results}

\section{MR lung perfusion parameter}

The reduction of the lung perfusion parameter can be observed already visually and qualitatively (Fig. 1).

Lung volume is significantly decreased on the ipsilateral side compared to the contralateral $(p=0.0012$; Table 1$)$. Lung perfusion parameters are significantly lower on the affected side than the contralateral, for example, mean
Fig. 1 Ten-year-old patient after $\mathrm{CDH}$ repair on the left side. Left: Coronal T2-weighted image. Right: Pulmonary blood flow map. Already visually, reduced perfusion is recognized on the ipsilateral side. Color scale on the right codes perfusion values $(\mathrm{mL} / 100 \mathrm{~mL} / \mathrm{min})$

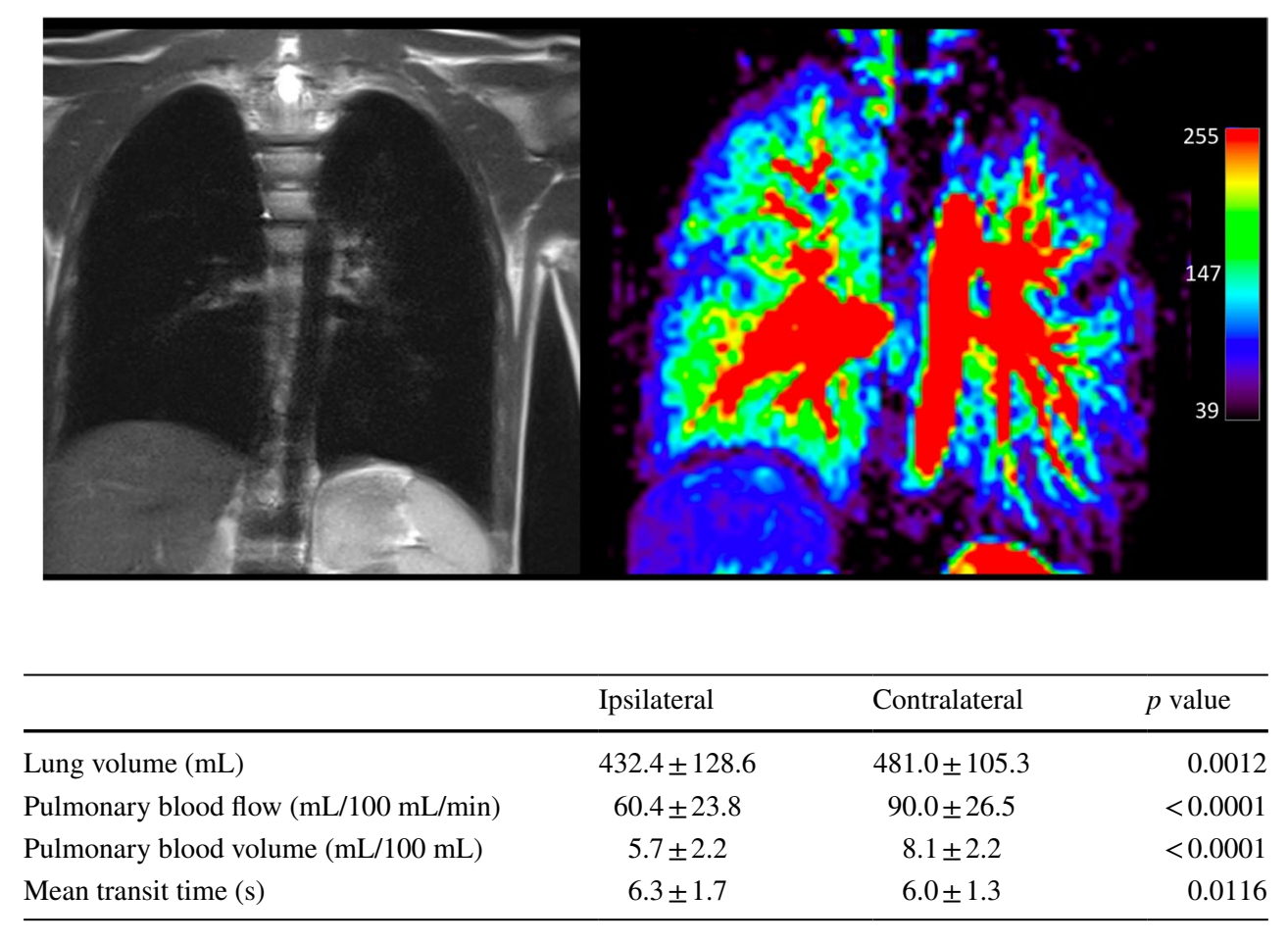

\begin{tabular}{lccr}
\hline & Ipsilateral & Contralateral & \multicolumn{1}{c}{$p$ value } \\
\hline Lung volume $(\mathrm{mL})$ & $432.4 \pm 128.6$ & $481.0 \pm 105.3$ & 0.0012 \\
Pulmonary blood flow $(\mathrm{mL} / 100 \mathrm{~mL} / \mathrm{min})$ & $60.4 \pm 23.8$ & $90.0 \pm 26.5$ & $<0.0001$ \\
Pulmonary blood volume $(\mathrm{mL} / 100 \mathrm{~mL})$ & $5.7 \pm 2.2$ & $8.1 \pm 2.2$ & $<0.0001$ \\
Mean transit time $(\mathrm{s})$ & $6.3 \pm 1.7$ & $6.0 \pm 1.3$ & 0.0116 \\
\hline
\end{tabular}

Table 1 Comparison of lung volume and lung perfusion parameters between contralateral and ipsilateral lung in $\mathrm{CDH}$ patients 
Fig. 2 Comparison of pulmonary blood flow in 10 -year-old $\mathrm{CDH}$ children with and without ECMO requirement

Table 2 Comparison of lung perfusion parameters between $\mathrm{CDH}$ patients and control group
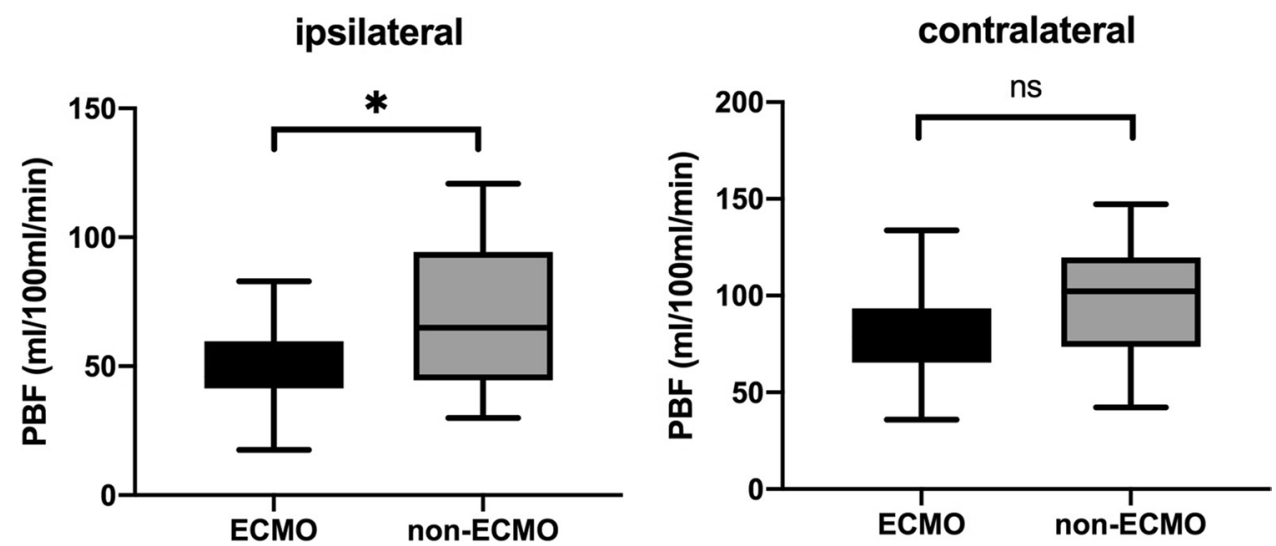

\begin{tabular}{lccc}
\hline & CDH & Controls* & $p$ value \\
\hline Lung volume ipsilateral & $432.4 \pm 128.6$ & $462.3 \pm 159.1$ & 0.5207 \\
Lung volume contralateral & $481.0 \pm 105.4$ & $480.4 \pm 160.2$ & 0.9892 \\
PBF ipsilateral $(\mathrm{mL} / 100 \mathrm{~mL} / \mathrm{min})$ & $60.4 \pm 23.8$ & $93.3 \pm 16.09$ & $0.0002^{*}$ \\
PBF contralateral $(\mathrm{mL} / 100 \mathrm{~mL} / \mathrm{min})$ & $90.0 \pm 26.5$ & $95.4 \pm 13.5$ & 0.5326 \\
PBF ratio (ipsi/contra) & $0.669 \pm 0.152$ & $0.975 \pm 0.091$ & $<0.0001^{*}$ \\
PBV ipsilateral $(\mathrm{mL} / 100 \mathrm{~mL})$ & $5.69 \pm 2.19$ & $7.92 \pm 2.65$ & $0.0128^{*}$ \\
PBV contralateral $(\mathrm{mL} / 100 \mathrm{~mL})$ & $8.08 \pm 2.18$ & $8.62 \pm 2.00$ & 0.4816 \\
MTT ipsilateral $(\mathrm{s})$ & $6.33 \pm 1.65$ & $5.82 \pm 0.95$ & 0.5442 \\
MTT contralateral $(\mathrm{s})$ & $5.95 \pm 1.28$ & $5.86 \pm 0.77$ & 0.7673 \\
\hline
\end{tabular}

$C D H$, congenital diaphragmatic hernia; $P B F$, pulmonary blood flow; $P B V$, pulmonary blood volume; $M T T$, mean transit time

*Ipsilateral in control group means left, contralateral right lung

ipsilateral $\mathrm{PBF}$ is $60.4 \mathrm{~mL} / 100 / \mathrm{mL} / \mathrm{min}$ compared to $90.0 \mathrm{~mL} / 100 / \mathrm{mL} / \mathrm{min}(p<0.0001$; Table 1$)$.

Ipsilateral pulmonary blood flow was significantly decreased in the ECMO group compared to children who did not require ECMO neonatally $(49.7 \pm 14.3$ vs. $67.8 \pm 26.1 \mathrm{~mL} / 100 \mathrm{~mL} / \mathrm{min} ; p=0.0056$; Fig. 2). In the contralateral lungs, there is no significant difference in perfusion parameters between the ECMO and the non-ECMO groups $(p=0.2420)$.

Pulmonary blood flow and pulmonary blood volume measurements were significantly lower on the ipsilateral side in children after congenital diaphragmatic hernia than those in normal controls (Table 2). The PBF ratio between ipsilateral and contralateral lung was close to 1 in the control group $(0.975 \pm 0.091)$ and accordingly lower in the $\mathrm{CDH}$ group $(0.669 \pm 0.152 ; p<0.0001)$ (Fig. 3).

\section{Lung function}

Lung function measurements were available in 43 children after CDH. Measurements showed that 17 (40\%) patients suffered from restrictive respiratory disorder, 3 children (7\%) from obstructive disorder, and 7 patients (16\%) from a

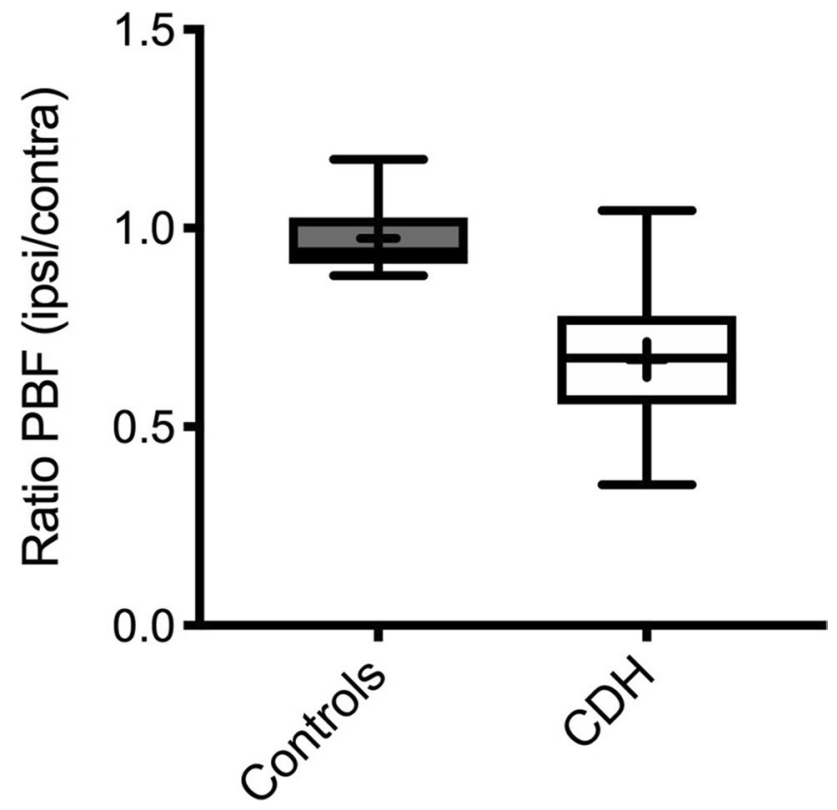

Fig. 3 Comparative whiskers plot of pulmonary blood flow ratio (ipsilateral/contralateral) of $\mathrm{CDH}$ children and the control group 
combination of both (Fig. 4). Sixteen (37\%) patients showed normal lung function parameters. The mean forced expiratory volume for all children was $75.98 \pm 22.82 \%$ of expected, and therefore lower than that in a healthy cohort. Forced vital capacity was also reduced with $74.79 \pm 20.69 \%$ of expected.

A comparison of children with and without ECMO requirement showed that respiratory disorder was more frequently present in children with ECMO requirement (82\%) than that in children without ECMO requirement (52\%; Fig. 4).

\section{Correlation between MR lung perfusion parameter and lung function}

Ipsilateral PBF was significantly lower in children with respiratory disorder $(53.8 \pm 16.1 \mathrm{~mL} / 100 \mathrm{~mL} / \mathrm{min})$ in comparison to that in children without respiratory disorder $(70.9 \pm 27.9 \mathrm{~mL} / 100 \mathrm{~mL} / \mathrm{min} ; p=0.0160$; Fig. 5). Contralateral PBF showed no significant difference between children with and without respiratory disorder $(85.4 \pm 24.4$ vs. $98.1 \pm 27.9 \mathrm{~mL} / 100 \mathrm{~mL} / \mathrm{min} ; p=0.1272$ ).

There was a significant positive correlation between PBF ipsilateral and forced vital capacity (FVC; $r=0.39$; $p=0.0091$; Fig. 6) and forced expiratory volume (FEV1; $r=0.45 ; p=0.0024$, Fig. 6 ).

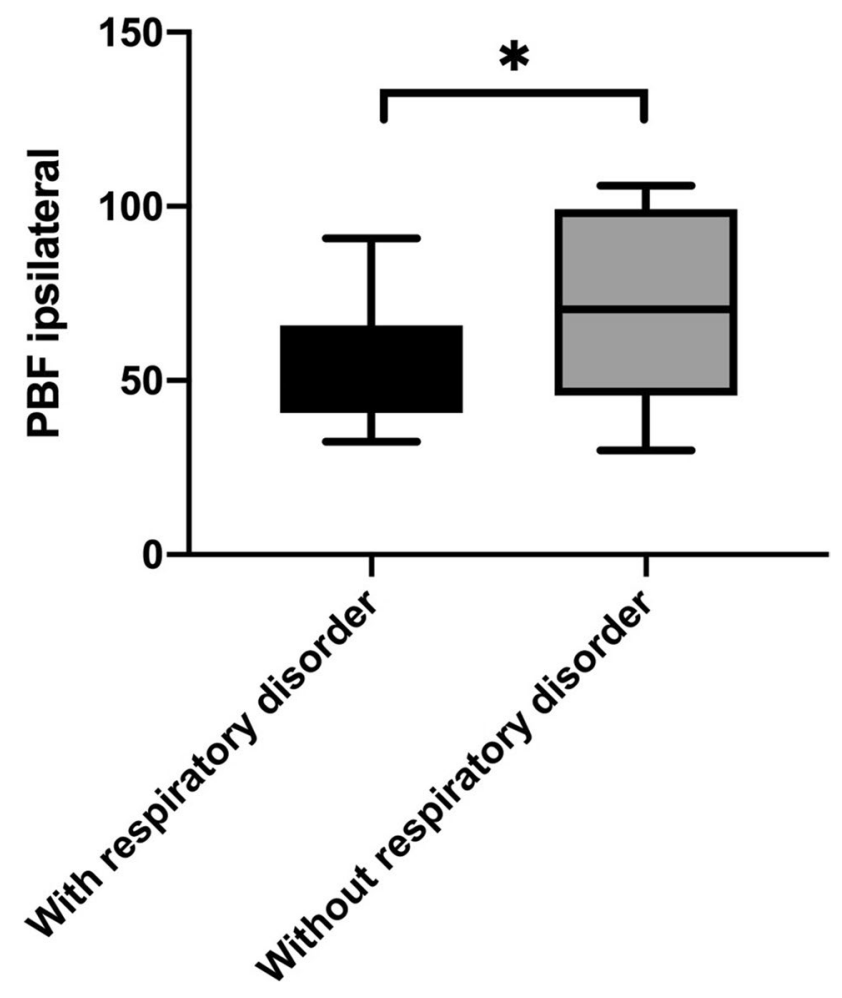

Fig. 5 Difference of pulmonary blood flow between children with and without respiratory disorder

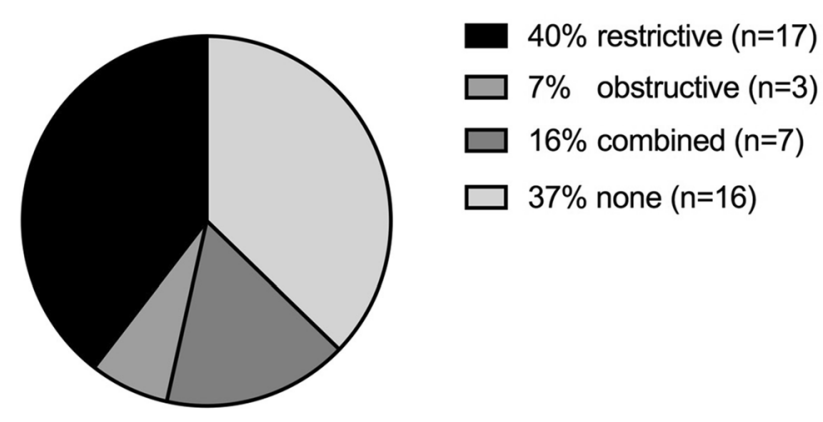

Total $=43$

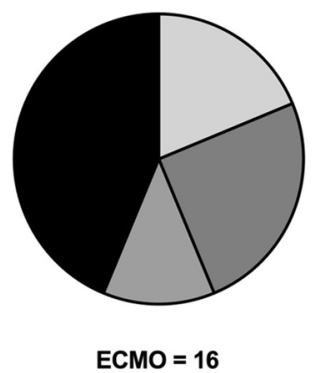

$44 \%$ restrictive $(n=7)$

$\square 13 \%$ obstructive $(n=2)$

$\square 25 \%$ combined $(n=4)$

$\square 18 \%$ none $(n=3)$

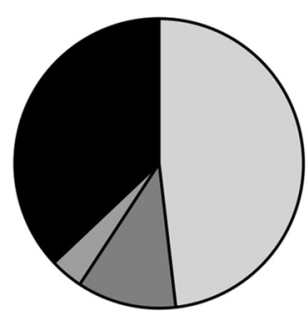

$37 \%$ restrictive $(n=10)$

$4 \%$ obstructive $(n=1)$

$\square 11 \%$ combined $(n=3)$

$48 \%$ none $(n=13)$

Fig. 4 Distributions of respiratory disorders. On the left side, distribution of all children demonstrated. On the right side, distribution of ECMO and non-ECMO children is visualized 

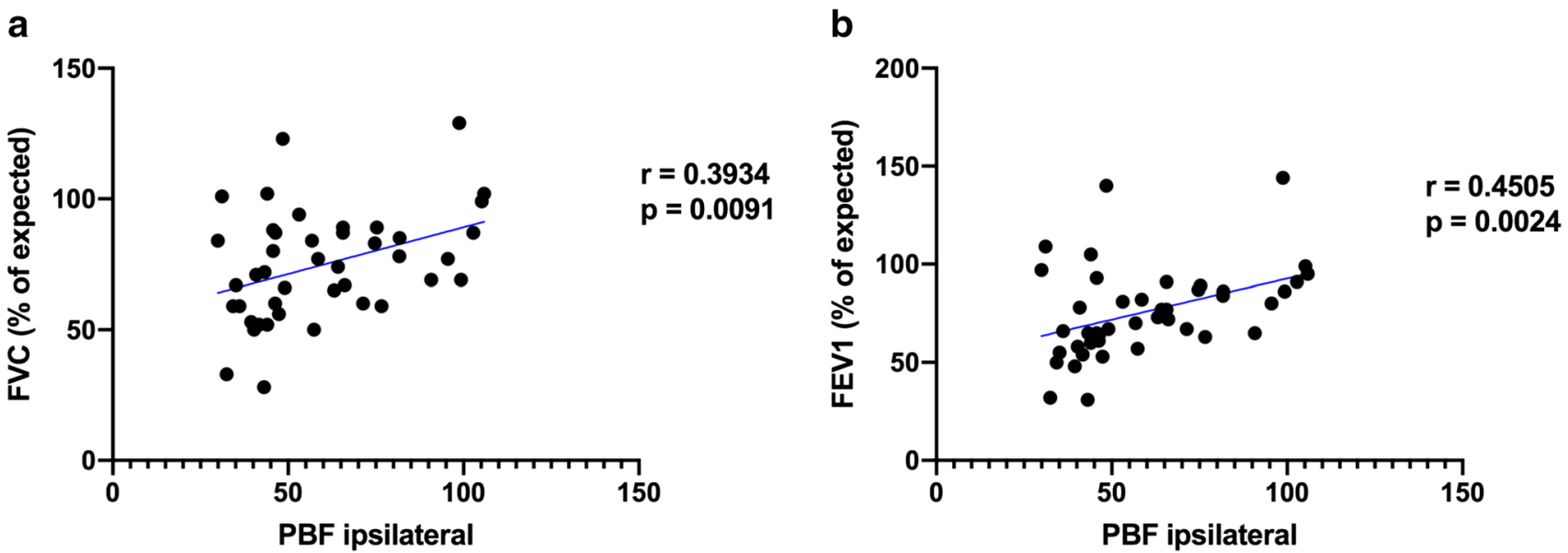

Fig. 6 Correlation of pulmonary blood flow with spirometric results. a There is a positive correlation between ipsilateral pulmonary blood flow (PBF) and forced vital capacity (FVC). b There is as well a positive correlation between PBF and forced expiratory 1-s volume (FEV1)

\section{Discussion}

Adolescent children (10-12 years) after congenital diaphragmatic hernia $(\mathrm{CDH})$ show reduced lung perfusion values ipsilateral, which can be detected by magnetic resonance imaging.

This difference in lung perfusion has been demonstrated previously in 2-year-old children after CDH [10]. In comparison to former studies, now older children have been examined. The present study demonstrates that ipsilateral perfusion deficits persist during childhood. According to present study, mean ipsilateral pulmonary blood flow (PBF) in 10-year-old children is $60 \mathrm{~mL} / 100 / \mathrm{mL} / \mathrm{min}$ and therefore similar to published values of $62 \mathrm{~mL} / 100 \mathrm{~mL} / \mathrm{min}$ in 2-yearolds [5]. The ratio of ipsi- and contralateral pulmonary blood flow is also comparable between 2-year-old children (0.69; [5]) and 10-12-year-old patients (0.67). Both the present and the previous study are based on cross-sectional data of 2-year- and 10-12-year-old children, respectively. Therefore, a direct comparison of absolute values is difficult. Future studies will need to address the individual course of lung perfusion longitudinally.

In accordance with other studies, the PBF ratio (ipsilateral/contralateral) of children after $\mathrm{CDH}$ differs significantly from the PBF ratio in healthy lungs, which is close to $1[11$, 12]. Therefore, the PBF ratio of ipsilateral to contralateral lung could serve as a parameter to grade the degree of lung perfusion impairment.

As demonstrated for 2-year-old children [6], children who required extracorporeal membrane oxygenation (ECMO) therapy in their neonatal period show even more reduced lung perfusion values on the ipsilateral side. ECMO therapy is only performed in case of respiratory or hemodynamic failure associated with severe pulmonary hypertension; and therefore, status after ECMO is a sign of CDH severity. The current study demonstrates that structural changes in vascular bed —represented by lung perfusion —are not regenerated during childhood but persist until the age of adolescence. This result is in accordance with literature demonstrating that children after ECMO therapy suffer from higher morbidity [13-16].

Scintigraphical measurements have also depicted perfusion deficits. In scintigraphy, lower ventilation to perfusion coefficients are present. Bjorkman et al. showed that 6-month-old infants have a variable degree of ventilationperfusion abnormalities, which are correlated with the persistence of pulmonary hypertension [17]. Stefanutti et al. investigated several outcome parameters in 8-year-old $\mathrm{CDH}$ patients. Among others, they found reduced perfusion values on the ipsilateral side [18]. Hayward et al. investigated changes in ventilation/perfusion (V/Q) mismatch during childhood in serial scintigraphical measurements: They could demonstrate that some survivors develop severe V/Q mismatch during childhood [19] — these children are at risk for severe pulmonary morbidity and need intensive follow-up. In our study, we could also demonstrate that perfusion deficits, which could be depicted in 2-year-old children, persist until the age of 10. However, intraindividual changes have not been evaluated yet. Pal et al. evaluated serial perfusion studies at the ages of 3 months, 9 months, and 6 years [20]. Thereby, they found that lung perfusion is reduced in the ipsilateral lung but increases during childhood, associated with amelioration of lung function parameters. In contrast to scintigraphical methods, MR perfusion measurements are feasible without ionizing radiation and seem promising as a follow-up tool. Future studies are needed to evaluate the individual course of lung perfusion, measured by MRI. A promising development in this context is the implementation 
of perfusion measurements without the need for a contrast agent. One technique, which has already been used to study pulmonary perfusion, is the Fourier decomposition technique $[21,22]$. Recently, a further development of this technique, has been used in patients after cystic fibrosis [23, 24]. To our knowledge, a transfer of this technique to younger patients with associated technical challenges has not been performed yet but seems promising.

Lung perfusion measurements have also been performed intrauterine by sonographic measurements. Thereby, it could be demonstrated that children with $\mathrm{CDH}$ show reduced lung perfusion values associated with increased pulmonary artery resistance already prenatally [25]. In combination with the present study, these results show that a hypoplastic or dysfunctional vascular bed is one key in the further understanding of morbidity in congenital diaphragmatic hernia.

Another-or a related-part of morbidity after $\mathrm{CDH}$ is the presence of lung function morbidity. The current study demonstrates that adolescent children after $\mathrm{CDH}$ suffer in around $40 \%$ from restrictive, in $16 \%$ from combined, and in $7 \%$ from obstructive lung function impairment. A total of $37 \%$ have average spirometric results. Both forced expiratory volume (FEV) and forced vital capacity (FVC) were reduced in the study cohort. These results are in good agreement with a study of Majaesic et al., which also demonstrates reduced FEV and FVC values in 8-year-old children after CDH [26].

Similarly, a study that investigated 13-year-old children also showed (besides others) reduced values of FEV [27, 28]. Interestingly, an investigation of 24-year-old CDH survivors could demonstrate reduced FEV values in some survivors, but this was not associated with reduced quality of life [29]. This is in agreement with the results of Arena et al.—despite persistent measurable FEV deficits, symptoms of obstruction disappeared [30]. These results raise, of course, the question of whether persistent airway flow impairment measurements are accompanied by respiratory problems later in life, for example, in their forties. This question cannot be answered yet. During early childhood, CDH survivors suffer from recurrent respiratory tract infections and have a higher risk of developing asthma [28, 31]. In our study cohort, significant differences between $\mathrm{CDH}$ patients with neonatal ECMO therapy as compared to those without ECMO therapy could be detected concerning severity of ipsilateral perfusion deficits and respiratory disorders.

The current study shows a relationship between ipsilateral pulmonary blood flow and both expected FEV1 and expected FVC. Therefore, MR perfusion values correlate with respiratory measurements. On the one hand, this can be explained by structural lung changes of vascular bed and airways that persist during childhood [32]. On the other hand, lung ventilation and perfusion are associated with several mechanisms, of which the Euler-Liljestrand is the most popular [33].
One limitation of this study is the restricted number of patients and normal controls, which is mainly owed by retrospective study design. Another limitation of this study is the manual placement of arterial input function and manual lung delineation-future studies should help transfer automatic lung segmentation, which have been proposed by several authors $[34,35]$ to clinical routine. Another limitation of the present study is that the comparison of mean perfusion values per lung side eliminates the possibility of comparing regional differences or distribution patterns. Future studies should address this question potentially by applying histogram-based analyses.

To summarize, MR lung perfusion deficits persist at least until the age of 10 years after $\mathrm{CDH}$. MR perfusion measurements correlate to lung function measurements without the need for compliance during measurement. Therefore, MR perfusion measurement could be a tool for stratifying the need for more intensive or longer follow-up in children after $\mathrm{CDH}$ without ionizing radiation. This would be of advantage especially for high-risk patients with $\mathrm{CDH}$ requiring neonatal ECMO therapy.

Funding Open Access funding enabled and organized by Projekt DEAL. This study has received funding by Deutsche Forschungsgemeinschaft (DFG, German Research Foundation) - 397806429.

\section{Declarations}

Guarantor The scientific guarantor of this publication is PD Dr.med. Meike Weis, MSc.

Conflict of interest The authors of this manuscript declare no relationships with any companies whose products or services may be related to the subject matter of the article.

Statistics and biometry One of the authors has significant statistical expertise (Prof. Dr. Christel Weiß).

Informed consent Written informed consent was obtained from all subjects (patients) in this study.

Ethical approval Institutional Review Board approval was obtained.

Study subjects or cohorts overlap Fifteen patients of our current cohort (54 patients) have been examined at the age of 2 years. Four of them with identical study protocol. Now, they have been re-examined at the age of 10 years.

\section{Methodology \\ - retrospective \\ - diagnostic or prognostic study \\ - performed at one institution}

Open Access This article is licensed under a Creative Commons Attribution 4.0 International License, which permits use, sharing, adaptation, distribution and reproduction in any medium or format, as long as you give appropriate credit to the original author(s) and the source, 
provide a link to the Creative Commons licence, and indicate if changes were made. The images or other third party material in this article are included in the article's Creative Commons licence, unless indicated otherwise in a credit line to the material. If material is not included in the article's Creative Commons licence and your intended use is not permitted by statutory regulation or exceeds the permitted use, you will need to obtain permission directly from the copyright holder. To view a copy of this licence, visit http://creativecommons.org/licenses/by/4.0/.

\section{References}

1. Snoek KG, Reiss IK, Greenough A et al (2016) Standardized postnatal management of infants with congenital diaphragmatic hernia in Europe: The CDH EURO Consortium Consensus - 2015 Update. Neonatology 110:66-74

2. IJsselstijn H, Breatnach C, Hoskote A et al (2018) Defining outcomes following congenital diaphragmatic hernia using standardised clinical assessment and management plan (SCAMP) methodology within the CDH EURO consortium. Pediatr Res 84:181-189

3. Hollinger LE, Buchmiller TL (2020) Long term follow-up in congenital diaphragmatic hernia. Semin Perinatol 44:151171

4. Kirby E, Keijzer R (2020) Congenital diaphragmatic hernia: current management strategies from antenatal diagnosis to long-term follow-up. Pediatr Surg Int 36:415-429

5. Weis M, Sommer V, Zöllner FG et al (2016) Region of interestbased versus whole-lung segmentation-based approach for MR lung perfusion quantification in 2-year-old children after congenital diaphragmatic hernia repair. Eur Radiol 26:4231-4238

6. Weis M, Zoellner FG, Hagelstein C et al (2016) Lung perfusion MRI after congenital diaphragmatic hernia repair in 2-year-old children with and without extracorporeal membrane oxygenation therapy. AJR Am J Roentgenol 206:1315-1320

7. Zoellner FG, Zahn K, Schaible T, Schoenberg SO, Schad LR, Neff KW (2012) Quantitative pulmonary perfusion imaging at 3.0 T of 2-year-old children after congenital diaphragmatic hernia repair: initial results. Eur Radiol 22:2743-2749

8. Zoellner FG, Weisser G, Reich M et al (2013) UMMPerfusion: an open source software tool towards quantitative MRI perfusion analysis in clinical routine. J Digit Imaging 26:344-352

9. Zöllner FG, Daab M, Sourbron SP, Schad LR, Schoenberg SO, Weisser G (2016) An open source software for analysis of dynamic contrast enhanced magnetic resonance images: UMMPerfusion revisited. BMC Med Imaging 16:7

10. Weidner M, Zoellner FG, Hagelstein C et al (2014) High temporal versus high spatial resolution in MR quantitative pulmonary perfusion imaging of two-year old children after congenital diaphragmatic hernia repair. Eur Radiol 24:2427-2434

11. Ley S, Ley-Zaporozhan J (2012) Pulmonary perfusion imaging using MRI: clinical application. Insights Imaging 3:61-71

12. Nael K, Michaely HJ, Lee M, Goldin J, Laub G, Finn JP (2006) Dynamic pulmonary perfusion and flow quantification with MR imaging, 3.0T vs. 1.5T: initial results. J Magn Reson Imaging 24:333-339

13. Ijsselstijn H, van Heijst AF (2014) Long-term outcome of children treated with neonatal extracorporeal membrane oxygenation: increasing problems with increasing age. Semin Perinatol 38:114-121

14. Madderom MJ, Reuser JJ, Utens EM et al (2013) Neurodevelopmental, educational and behavioral outcome at 8 years after neonatal ECMO: a nationwide multicenter study. Intensive Care Med 39:1584-1593

15. Madderom MJ, Toussaint L, van der Cammen-van Zijp MHM et al (2012) Congenital diaphragmatic hernia with(out) ECMO: impaired development at 8 years. Arch Dis Child Fetal Neonatal Ed. https://doi.org/10.1136/archdischild-2012-303020

16. Stolar CJ, Crisafi MA, Driscoll YT (1995) Neurocognitive outcome for neonates treated with extracorporeal membrane oxygenation: are infants with congenital diaphragmatic hernia different? J Pediatr Surg 30:366-371; discussion 371-362

17. Bjorkman KC, Kjellberg M, Bergstrom SE et al (2011) Postoperative regional distribution of pulmonary ventilation and perfusion in infants with congenital diaphragmatic hernia. J Pediatr Surg 46:2047-2053

18. Stefanutti G, Filippone M, Tommasoni N et al (2004) Cardiopulmonary anatomy and function in long-term survivors of mild to moderate congenital diaphragmatic hernia. J Pediatr Surg 39:526-531

19. Hayward MJ, Kharasch V, Sheils C et al (2007) Predicting inadequate long-term lung development in children with congenital diaphragmatic hernia: an analysis of longitudinal changes in ventilation and perfusion. J Pediatr Surg 42:112-116

20. Pal K, Gupta DK (2010) Serial perfusion study depicts pulmonary vascular growth in the survivors of non-extracorporeal membrane oxygenation-treated congenital diaphragmatic hernia. Neonatology 98:254-259

21. Bondesson D, Schneider MJ, Gaass T et al (2019) Nonuniform Fourier-decomposition MRI for ventilation- and perfusionweighted imaging of the lung. Magn Reson Med 82:1312-1321

22. Lederlin M, Bauman G, Eichinger M et al (2013) Functional MRI using Fourier decomposition of lung signal: reproducibility of ventilation- and perfusion-weighted imaging in healthy volunteers. Eur J Radiol 82:1015-1022

23. Kunz AS, Weng AM, Wech T et al (2021) Non-contrast pulmonary perfusion MRI in patients with cystic fibrosis. Eur J Radiol 139:109653

24. Veldhoen S, Weng AM, Knapp J et al (2017) Self-gated noncontrast-enhanced functional lung MR imaging for quantitative ventilation assessment in patients with cystic fibrosis. Radiology 283:242-251

25. Moreno-Alvarez O, Cruz-Martinez R, Hernandez-Andrade E et al (2010) Lung tissue perfusion in congenital diaphragmatic hernia and association with the lung-to-head ratio and intrapulmonary artery pulsed Doppler. Ultrasound Obstet Gynecol 35:578-582

26. Majaesic CM, Jones R, Dinu IA, Montgomery MD, Sauve RS, Robertson CM (2007) Clinical correlations and pulmonary function at 8 years of age after severe neonatal respiratory failure. Pediatr Pulmonol 42:829-837

27. Haliburton B, Mouzaki M, Chiang M et al (2017) Pulmonary function and nutritional morbidity in children and adolescents with congenital diaphragmatic hernia. J Pediatr Surg 52:252-256

28. Öst E, Joelsson M, Burgos CM, Frenckner B (2016) Self-assessed physical health among children with congenital diaphragmatic hernia. Pediatr Surg Int 32:493-503

29. Peetsold MG, Vonk-Noordegraaf A, Heij HH, Gemke RJ (2007) Pulmonary function and exercise testing in adult survivors of congenital diaphragmatic hernia. Pediatr Pulmonol 42:325-331

30. Arena F, Baldari S, Centorrino A et al (2005) Mid- and long-term effects on pulmonary perfusion, anatomy and diaphragmatic motility in survivors of congenital diaphragmatic hernia. Pediatr Surg Int 21:954-959 
31. Rocha G, Azevedo I, Pinto JC, Guimarães H (2012) Follow-up of the survivors of congenital diaphragmatic hernia. Early Hum Dev $88: 255-258$

32. Waag KL, Loff S, Zahn K et al (2008) Congenital diaphragmatic hernia: a modern day approach. Semin Pediatr Surg 17:244-254

33. Sommer N, Strielkov I, Pak O, Weissmann N (2016) Oxygen sensing and signal transduction in hypoxic pulmonary vasoconstriction. Eur Respir J 47:288-303

34. Mueller J, Karrasch S, Lorbeer R et al (2019) Automated MRbased lung volume segmentation in population-based wholebody MR imaging: correlation with clinical characteristics, pulmonary function testing and obstructive lung disease. Eur Radiol 29:1595-1606
35. Zoellner FG, Daab M, Weidner M et al (2015) Semi-automatic lung segmentation of DCE-MRI data sets of 2-year old children after congenital diaphragmatic hernia repair: Initial results. Magn Reson Imaging 33:1345-1349

Publisher's note Springer Nature remains neutral with regard to jurisdictional claims in published maps and institutional affiliations. 